\title{
Optofluidic On-Chip Tomography
}

\author{
Serhan O. Isikman, Student Member, IEEE, Waheb Bishara, Hongying Zhu and Aydogan Ozcan, \\ Senior Member, IEEE
}

\begin{abstract}
The first demonstration of optofluidic tomography is presented. Using partially coherent illumination, holograms of objects are recorded at multiple viewing angles, as they flow through a microfluidic channel placed directly on the top of an opto-electronic sensor array. These lensfree holograms are then digitally processed to compute pixel super-resolved tomograms of micro-objects to achieve sectional opto-fluidic imaging on a chip.
\end{abstract}

\section{INTRODUCTION}

$\mathrm{T}$ he combination of optics and microfluidics gave rise to the field of optofluidics, which is a powerful integrated platform for lab-on-a-chip systems [1],[2]. Adaptive-optical lenses [3], flow-cytometers [4], bio-sensors [5]-[8] and tunable lasers [9],[10] are among the recently developed optofluidic systems. An exciting direction in optofluidics is towards the development of on-chip imaging modalities. Recently introduced optofluidic microscopy platforms [11],[12] offer high-throughput handling and imaging of micro-objects in a compact and versatile architecture.

In addition to these, there have been significant developments in microscopy modalities that can achieve sectional imaging. Optical projection tomography [13],[14], optical diffraction tomography [15]-[19] and light-sheet microscopy [20],[21] techniques have been developed for microscopic imaging of objects such as cells and multicellular model organisms. Nevertheless, an on-chip 3D imaging platform that can be integrated with lab-on-a-chip devices has not yet been introduced. Towards this end, we present the first demonstration of optofluidic tomography, which can perform sectional imaging of objects flowing through a microfluidic channel on-a-chip. Optofluidic tomography, with its decent 3D spatial resolution, could provide a new toolset for various biomedical imaging applications that utilize lab-on-a-chip devices.

\section{OPTOFLUIDIC TOMOGRAPHY SET-UP}

Our optofluidic tomography platform builds on partially coherent lensfree on-chip holography [12],[22]. As illustrated in Fig. 1, a partially coherent light source $(\sim 10 \mathrm{~nm}$ around a center wavelength of $600 \mathrm{~nm})$ is used to

S. O. I., W. B., and H. Z are with the Electrical Engineering Department at University of California, Los Angeles, CA, 90095, USA (S. O. I. e-mail: sisikman@ucla.edu).

A. O. is with the Electrical Engineering Department at University of California, Los Angeles, CA, 90095, USA, and California NanoSystems Institute, Los Angeles, CA, 90095, USA (e-mail: ozcan@ucla.edu; http://innovate.ee.ucla.edu/). illuminate samples flowing through a microfluidic channel. The slanted microfluidic channel having a relatively large cross-section of $\sim 80 \mu \mathrm{m} \times 1 \mathrm{~mm}$ is placed directly on top of a digital sensor array (Aptina MT9P031STC, 5 Megapixels, $2.2 \mu \mathrm{m}$ pixel size) such that the objects flow at a distance of $<1 \mathrm{~mm}$ above the active area of the sensor-chip. While the objects are electro-kinetically driven through the microchannel, lensfree inline-holograms of the sample are recorded by varying the illumination direction within an angular range of $\pm 50^{\circ}$. In order to employ pixel superresolution (SR) techniques [12], multiple holograms for each illumination angle are recorded (while the objects are flowing within the channel), which enabled us to digitally synthesize a single SR hologram with increased the numerical aperture (NA $\sim 0.3-0.4$ ) for each viewing angle.

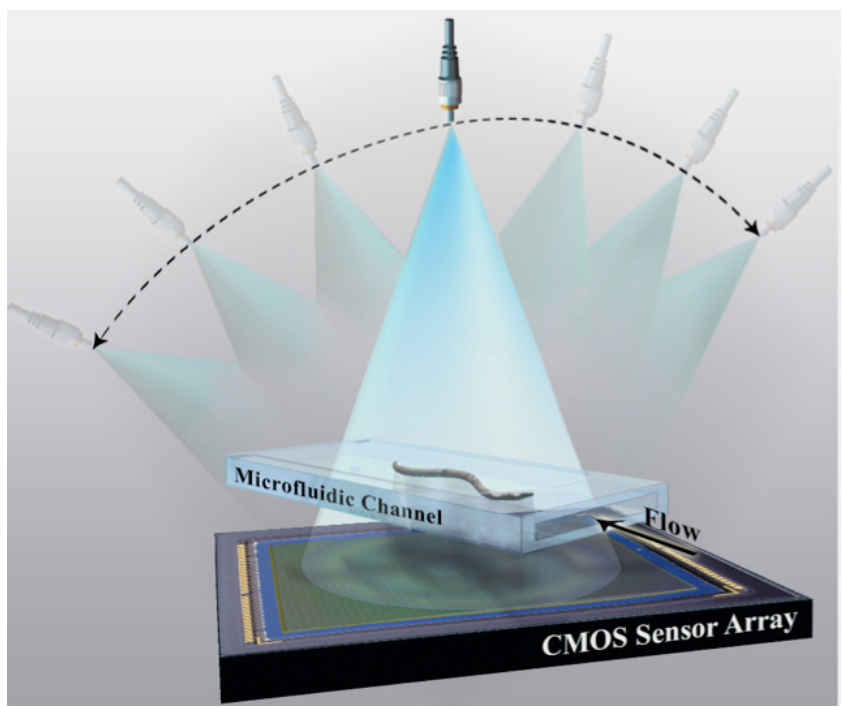

Fig. 1. Schematic illustration of the optofluidic tomographic microscope is shown. The objects are electro-kinetically driven through the slanted microfluidic channel, placed on the top of a digital sensor array, while partially-coherent illumination is utilized to record lensfree digital inline holograms at varying illumination angles.

\section{EXPERIMENTAL RESULTS}

In order to demonstrate the proof-of-concept of optofluidic tomography, we conducted experiments with a wild-type C. Elegans worm, which is a commonly used model organism in biomedical sciences. Multiple holograms of a C. Elegans worm at each illumination angle between $\pm 50^{\circ}$ with $2^{\circ}$ increments were recorded as the worm flowed rigidly through the microfluidic channel. The flow speed was maintained at approximately $1 \mu \mathrm{m} / \mathrm{sec}$ such that consecutively recorded holograms (with 5 frames/sec) at a given angle can be treated as shifted versions of the 
same hologram with respect to the pixel grid of the sensorarray. That is, in each frame recorded at a fixed illumination angle, the digital sensor array physically samples the same optical field with different sub-pixel shifts as the object rigidly flows within the channel. Therefore, pixel superresolution techniques can be employed to synthesize SR holograms [12] for each viewing angle using multiple lower resolution (LR) raw holograms measured for the corresponding illumination angle. Fig. 2 shows LR and SR holograms for three illumination angles, i.e. $-34^{\circ}, 0^{\circ}$ and $34^{\circ}$, where the aliased high-frequencies in the LR lensless holograms are clearly resolved in their corresponding SR holograms, thereby increasing the NA of our images. It should also be noted that a priori knowledge of the object shift is not necessary, and in fact these shifts are digitally estimated using an iterative gradient algorithm [12].
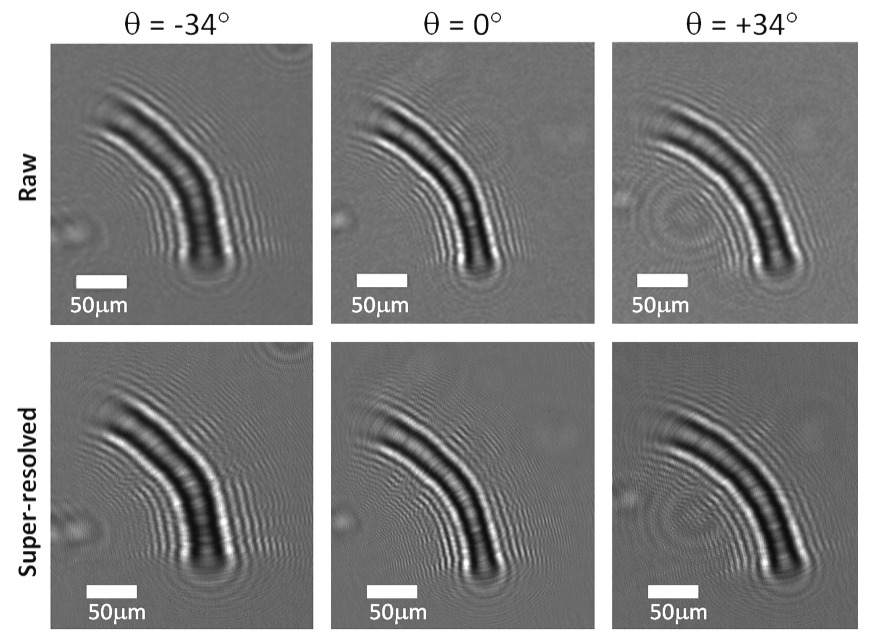

Fig. 2. (Top) Lensfree LR (raw) holograms of a C. elegans worm at three different illumination angles $\left(\theta=-34^{\circ}, 0^{\circ}\right.$ and $\left.34^{\circ}\right)$ are shown. (Bottom) SR (super-resolved) holograms for the same viewing angles are presented. Note that the lensfree holograms are wider for the tilted illumination angles, as expected, when compared to the vertical illumination case.
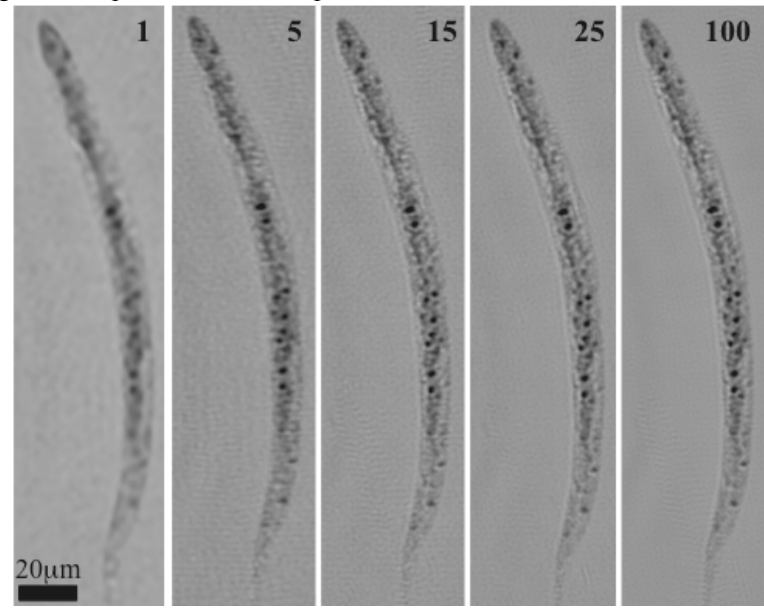

Fig. 3. Digitally reconstructed SR holograms (for the case of vertical illumination) are shown. The number on the top-right corner of each image denotes the number of consecutive frames used to synthesize the corresponding SR hologram. Based on these results, we concluded that recording $\sim 15$ frames (raw holograms) for each angle is sufficient to perform pixel super-resolution.
In order to determine the optimum number of frames to use per illumination angle, we reconstructed the SR holograms of an object calculated by using different number of frames ranging between 1 and 100. As shown in Fig. 3, $\sim 15$ frames are sufficient to achieve a decent image quality, and using more frames does not further increase the resolution.

Once a set of SR holograms for all illumination angles (total of 51 holograms) is digitally synthesized, lensfree images of the objects for these viewing angles can be obtained by a custom developed digital reconstruction algorithm.[22] Although oblique illumination is utilized during the recording process, each SR hologram is still an inline hologram. Therefore, as in conventional inline holographic microscopy, the recorded holograms are digitally reconstructed by multiplying with a digital reference wave, which in our case is a tilted plane wave with a propagation angle of $\theta$. We further use a supportconstrained iterative phase-recovery algorithm to eliminate the twin-image artifacts associated with inline holography. In $\sim 10-20$ iterations where the field is digitally propagated back-and-forth between detector and object planes, the complex optical field transmitted by the object for all illumination angles can be obtained. To demonstrate that, Fig. 4 shows digitally reconstructed lensfree amplitude and phase images of a $C$. elegans sample for different angles of illumination.

For weakly scattering objects, the recovered optical field can be assumed to be the projection of the 3D transmission function of the object [13]. Owing to the large depth-offocus of our digitally reconstructed lensfree images (e.g. $\sim 50 \mu \mathrm{m}$ ), which is a general property of inline holography [25],[26], this projection assumption is further satisfied since all points of the object lie within the depth-of-focus of our imaging platform. In this case, the reconstructed amplitude images, which we call lensfree projection images, can be back-projected to reconstruct the $3 \mathrm{D}$ transmission function of the objects. That is, using a weighted back-projection algorithm [27], tomographic images of the transmission function of the object can be obtained, permitting sectional imaging with significantly increased axial-resolution compared to a single in-line hologram. Fig. 5 shows tomograms computed for a $C$. Elegans worm. Distinct details can be observed at each slice shown in Fig. 5, which demonstrates optical sectioning with our optofluidic tomography platform. We also verified that that the axial full-width-at-half-maximum (FWHM) along the tomogram of the worm roughly matches with its actual thickness of $\sim 30 \mu \mathrm{m}$. The acquisition of this holographic data took $\sim 2.5 \mathrm{~min}$, which was limited by the frame rate of the digital sensor array ( 5 frames/sec). The acquisition time can be significantly reduced, e.g., down to $<30$ sec using commercially available faster sensors. The entire data processing (synthesizing SR holograms, holographic reconstruction and weighted back-projection) takes $<3.5 \mathrm{~min}$ using a single graphical processing unit 
(GPU), which could be completed much faster with multiple GPUs working in parallel.

$$
\theta=0 \quad \theta=-34
$$

$\theta=0$

$\theta=+34$
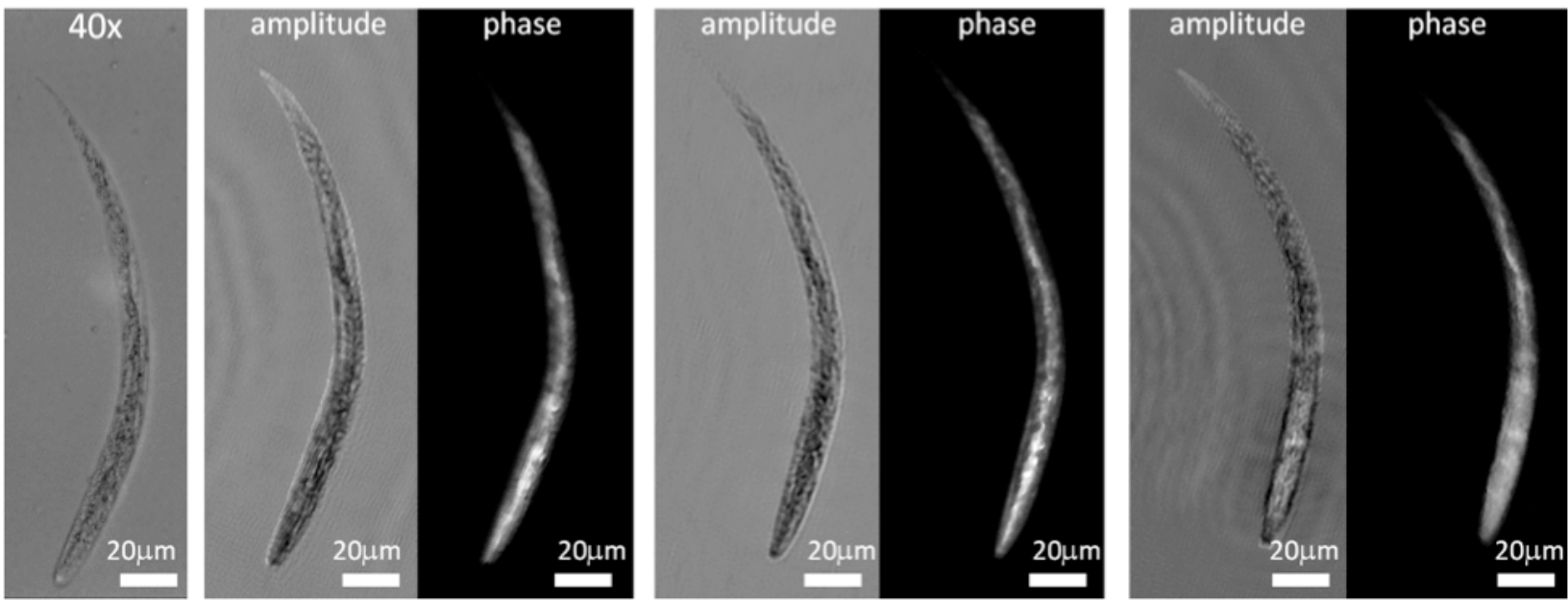

Fig. 4. Reconstructed amplitude and phase images for tilted illumination angles $\left(\theta=-34^{\circ}, 0^{\circ},+34^{\circ}\right)$ in optofluidic tomography are shown, along with a $40 \mathrm{X}$ objective-lens ( $0.65 \mathrm{NA}$ ) microscope comparison image (leftmost image) of the same C. Elegans worm.

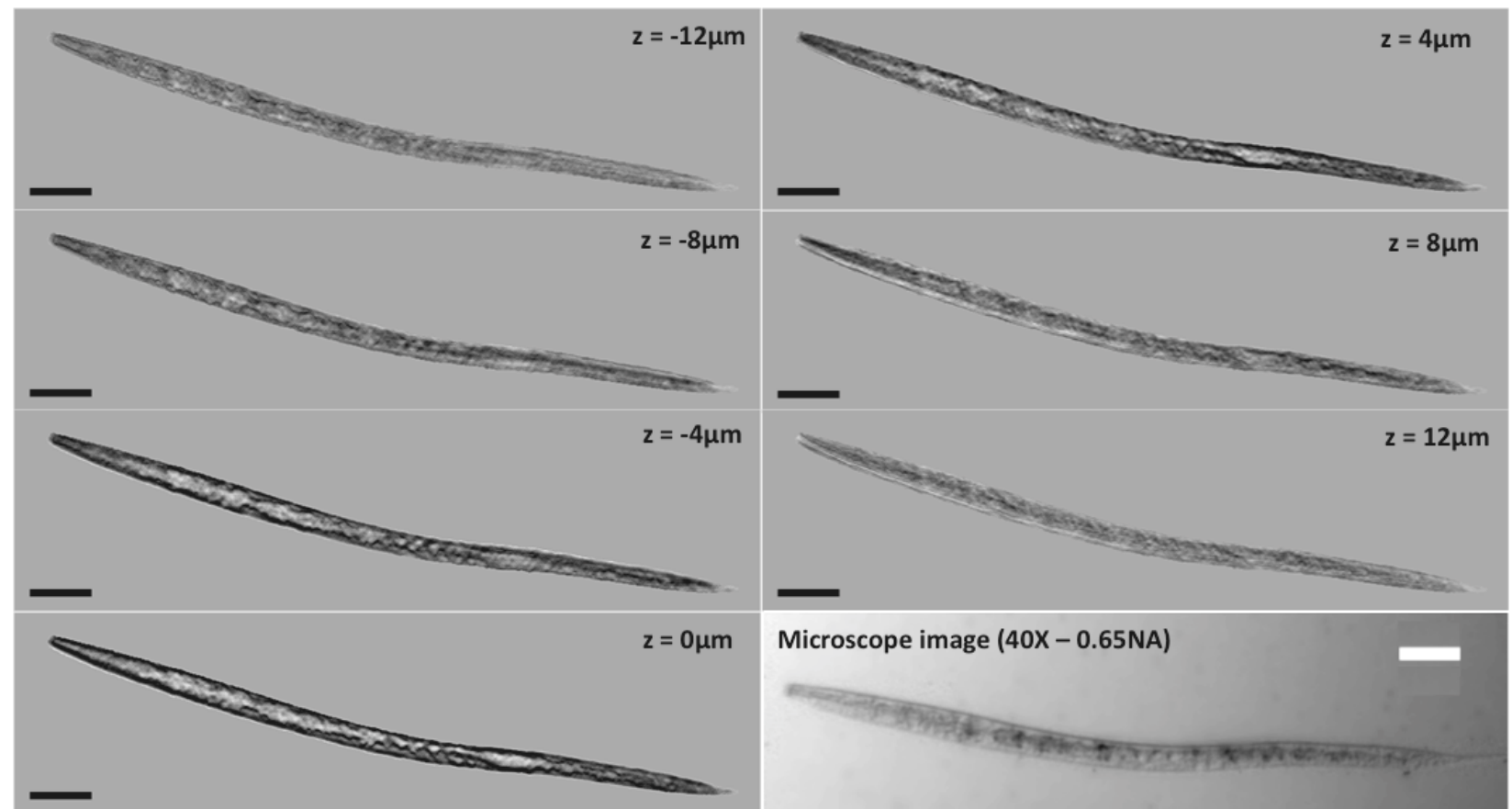

Fig. 5. Tomographic optofluidic imaging of $C$. Elegans is illustrated for different depth slices ( $\mathrm{z}=-12 \mu \mathrm{m}$ to $+12 \mu \mathrm{m})$. The FWHM of the amplitude of the line profile of the worm along $\mathrm{z}$ direction is calculated as $\sim 30 \mu \mathrm{m}$, which roughly agrees with its physical thickness. Scale bars: $50 \mu \mathrm{m}$

\section{DISCUSSION}

Our optofluidic tomography scheme provides a unique platform for achieving sectional imaging on a chip, which can be especially useful for 3D microscopy applications in microfluidic systems. We would like to emphasize that the lensfree hologram recording geometry with unit fringe magnification [12],[22] in our opto-fluidic microscopy platform conveniently permits imaging of flowing objects using various illumination angles as shown in Fig. 1, which is the key enabler for optofluidic tomography.

Whereas sub-micron lateral spatial resolution can be achieved through pixel super-resolution [12],[24], the axial resolution (along $\mathrm{z}$ ) is estimated to be $\sim 3 \mu \mathrm{m}$ since projection images are available for a limited range of angles, i.e. $\pm 50^{\circ}$, which theoretically results in a missing wedge in the Fourier space of the tomograms leading to an axially elongated point-spread function (PSF) [28]. Although not implemented in this work, the axial-resolution can be further improved by either implementing a dual-axis 
tomography scheme where projections are acquired along two orthogonal tilt directions [28],[29], or by implementing an iterative algorithm to digitally recover the missing region in the spatial-frequency spectrum of the object [30].

Acquiring the projection images through holographic recording and reconstruction provides a unique advantage to our optofluidic tomography platform to correct for possible fluctuations in the object's vertical position as it flows through the channel. Since projection holograms are digitally reconstructed at the best-focus distance, the object essentially does not go out-of-focus of the imaging system, and the change in the object's position within the channel does not result in significant artifacts in the computed tomograms. This is another important advantage of our holographic opto-fluidic tomography platform over conventional non-holographic opto-fluidic microscopes.

\section{ACKNOWLEDGMENT}

A. Ozcan gratefully acknowledges the support of NSF CAREER Award on BioPhotonics, the Office of Naval Research (ONR) under the Young Investigator Award and the NIH Director's New Innovator Award - Award Number DP2OD006427 from the Office of The Director, National Institutes of Health. The authors also acknowledge the support of the Bill-Melinda Gates Foundation, Vodafone Americas Foundation, NSF BISH program (under Awards \# 0754880 and 0930501), and AFOSR (under Project \# 08NE255).

\section{REFERENCES}

[1] D. Psaltis, S. R. Quake and C. Yang, "Developing optofluidic technology through the fusion of microfluidics and optics", Nature 442, 381 (2006)

[2] C. Monat, P. Domachuk and B. J. Eggleton, "Integrated optofluidics: A new river of light", Nature Photonics, 1, 106 (2007).

[3] L. Dong, A. K. Agarwal, D. J. Beebe and H. Jiang, "Adaptive liquid microlenses activated by stimuli-responsive hydrogels", Nature 442 , 551-554 (2006)

[4] J. Godin, C. H. Chen, S. H. Cho, W. Qiao, F. Tsai and Y.H. Lo, "Microfluidics and photonics for Bio-System-on-a-Chip: A review of advancements in technology towards a microfluidic flow cytometry chip", J. Biophoton. 1, 355 (2008)

[5] S. Mandal and D. Erickson, "Nanoscale optofluidic sensor arrays", Opt. Express 16, 1623 (2008)

[6] H. Shao, D. Kumar, K. L. Lear, "Single-Cell Detection Using Optofluidic Intracavity Spectroscopy”, IEEE Sensors Journal, vol.6, iss.6 (2006)

[7] P. Domachuk, I. C. M. Littler, M. C. Golomb, B. J. Eggleton, "Compact resonant integrated microfluidic refractometer", Appl. Phys. Lett., 88, 093513 (2006)

[8] A. D. Leebeck, L. K. S. Kumar, V. de Lange, D. Sinton, R. Gordon, A. G. Brolo, "On-Chip Surface-Based Detection with Nanohole Arrays", Anal. Chem., 79, 4094 (2007)

[9] W. Song and D. Psaltis, "Pneumatically tunable optofluidic dye laser", Appl. Phys. Lett. 96, 081101 (2010)

[10] Z. Li, D. Psaltis, "Optofluidic Distributed Feedback Dye Lasers", IEEE J. Sel. Top. In Quant. Elect., vol.13, no.2 (2007)

[11] X. Heng, D. Erickson, L. R. Baugh, Z. Yaqoob, P. W. Sternberg, D. Psaltis, C. Yang, "Optofluidic microscopy—a method for implementing a high resolution optical microscope on a chip", $L a b$ Chip 6, 1274 (2006)
[12] W. Bishara, H. Zhu, and A. Ozcan, "Holographic opto-fluidic microscopy", Optics Express, 18(26) (2010)

[13] J. Sharpe, U. Ahlgren, P. Perry, B. Hill, A. Ross, J. HecksherSørensen, R. Baldock and D. Davidson, "Optical Projection Tomography as a Tool for 3D Microscopy and Gene Expression Studies", Science, 296 (5567): 541-545

[14] M. Fauver, E. Seibel, J. R. Rahn, M. Meyer, F. Patten, T. Neumann, and A. Nelson, "Three-dimensional imaging of single isolated cell nuclei using optical projection tomography", Optics Express, Vol. 13, Issue 11, pp. 4210-4223 (2005)

[15] Y. Sung, W. Choi, C. Fang-Yen, K. Badizadegan, R. R. Dasari, and M. S. Feld, "Optical diffraction tomography for high resolution live cell imaging", Optics Express, Vol. 17, Issue 1, pp. 266-277 (2009)

[16] M. Debailleul, B. Simon, V. Georges, O. Haeberle, V. Lauer, "Holographic microscopy and diffractive microtomography of transparent samples", Meas. Sci. Technol. 19, 074009 (2008)

[17] M. Debailleul, V. Georges, B. Simon, R. Morin and O. Haeberlé, "High-resolution three-dimensional tomographic diffractive microscopy of transparent inorganic and biological samples", Opt. Lett., 34 (2009)

[18] K. Belkebir, P. C. Chaumet, and A. Sentenac, "Influence of multiple scattering on three-dimensional imaging with optical diffraction tomography", J. Opt. Soc. Am. A, Vol. 23, No. 3 (2006)

[19] F. Charrière, N. Pavillon, T. Colomb, C. Depeursinge, T. J. Heger, E. A. D. Mitchell, P. Marquet, B. Rappaz, "Living specimen tomography by digital holographic microscopy: morphometry of testate amoeba", Optics Express 14, 7005 (2006)

[20] J. Huisken, J. Swoger, F. DelBene, J. Wittbrodt and E. H. K. Stelzer, "Optical Sectioning Deep Inside Live Embryos by Selective Plane Illumination Microscopy" Science 305:1007-1009 (2004)

[21] P. J. Keller, A. D. Schmidt, J. Wittbrodt and E. H. K. Stelzer "Reconstruction of Zebrafish Early Embryonic Development by Scanned Light Sheet Microscopy", Science 322:1065-106 (2008)

[22] O. Mudanyali, D. Tseng, C. Oh, S.O. Isikman, I. Sencan, W. Bishara, C. Oztoprak, S. Seo, B. Khademhosseini, and A. Ozcan, "Compact, Light-weight and Cost-effective Microscope based on Lensless Incoherent Holography for Telemedicine Applications", Lab Chip, 10(11) 1417-1428 (2010)

[23] S. Seo, S.O. Isikman, I. Sencan, O. Mudanyali, T. Su, W. Bishara, A. Erlinger and A. Ozcan, "High-throughput Lensfree Blood Analysis On a Chip", Analytical Chemistry, 82(11) 4621-4627, (2010)

[24] W. Bishara, U. Sikora, O. Mudanyali, T. W. Su, O. Yaglidere, S. Luckhart and A. Ozcan, "Holographic pixel super-resolution in portable lensless on-chip microscopy using a fiber-optic array", $L a b$ Chip, 2011, 11, 1276-1279 (2011)

[25] H. Meng H and F. Hussain, "In-line recording and off-axis viewing technique for holographic particle velocimetry", Applied Optics 34:1827-1840 (2004)

[26] J. Sheng J, E. Malkiel and J. Katz J "Single Beam Two-Views Holographic Particle Image Velocimetry", Applied Optics 42:235250 (2003)

[27] M. Radermacher, "Weighted back-projection methods. Electron Tomography: Methods for three dimensional visualization of structures in the cell”, Springer, New York, 2nd ed. pp 245-273 (2006)

[28] D. N. Mastronarde, "Dual-Axis Tomography: An Approach with Alignment Methods That Preserve Resolution", Journal of Structural Biology 120:343-352 (1997)

[29] I. Arslan I, J. R. Tong, P. A. Midgley, "Reducing the missing wedge: High-resolution dual axis tomography of inorganic materials", Ultramicroscopy 106:994-1000 (2006)

[30] D. Verhoeven, "Limited-data computed tomography algorithms for the physical sciences", App. Optics 32:3736-3654 (1993) 